\title{
Excluded-volume polymer-induced depletion interaction between parallel flat plates
}

\author{
R. Tuinier ${ }^{\mathrm{a}}$ and H.N.W. Lekkerkerker \\ Van 't Hoff Laboratory, Debye Research Institute, University of Utrecht, Padualaan 8, NL-3584 CH Utrecht, The Netherlands
}

Received 9 August 2001

\begin{abstract}
The interaction between two parallel plates due to non-adsorbing polymer chains with excluded volume is calculated using the adsorption method. The adsorption is calculated from the profile of the polymer segment concentration between the plates, which is obtained from the product function of the concentration profile near a single wall, involving the correlation length. The renormalization group theory provides expressions for the osmotic pressure and consequently for the osmotic compressibility, chemical potential and correlation length of a polymer solution. Both the local polymer concentration profiles as well as the minimum of the interaction potential between the plates agree with recently published self-avoiding random walk computer simulations.
\end{abstract}

PACS. $61.25 . \mathrm{Hq}$ Macromolecular and polymer solutions; polymer melts; swelling - 82.70.Dd Colloids

\section{Introduction}

When non-adsorbing polymer chains are immersed in a suspension containing colloidal particles they impose an effective attractive interaction between those particles. This polymer-mediated depletion interaction has received significant attention in the last decades. Although the onset of the development of the depletion interaction was made by analysing the interaction between parallel plates by ideal chains [1], the description of the polymers was simplified even more by replacing the non-adsorbing polymers with penetrable hard spheres (PHS), sometimes also denoted as non-additive hard spheres. Penetrable hard spheres are hard spheres for the colloidal particles with which they interact but are permeable towards one another. The PHS concept was initiated by Vrij [2], who showed that it gives a rather accurate description of the depletion interaction due to ideal polymer chains. In fact, the minimum of the interaction potential of ideal polymers corresponds to that of PHSs if one takes $4 R_{\mathrm{g}} / \sqrt{\pi}$ as the effective PHS diameter [3], where $R_{\mathrm{g}}$ is the polymer's radius of gyration. The strength of the PHS simplification is that it allows a quantitative prediction of the consequences for the colloidal stability and the phase behaviour of colloidal dispersions as induced by non-adsorbing polymers. As a result, the PHS concept was used frequently over the last two decades during which theories focusing on the topology of the phase diagrams resulting from the PHS approach were developed $[4,5]$. Computer simulations $[6$, 7] (largely) confirmed these theoretical approaches.

\footnotetext{
a e-mail: r.tuinier@chem.uu.nl
}

The inclusion of the excluded-volume effects of the polymer segments has gained increasing attention [8-18] in order to bring the theoretical description closer to real polymer chains. Simplifications can be made in a successful way when relatively small colloidal spheres immersed in a polymer solution are considered [8-11], whereas a meanfield approach was developed for excluded-volume polymers between two plates [12]. For more complex systems, reference interaction site models (RISM) have been developed [13-16] that treat the polymers more realistically than PHS models do. Louis et al. [17] recently adopted a Gaussian core model for polymer chains that interact via a penetrable repulsive Gaussian potential. This model predicts the minimum of the interaction potential between two parallel flat plates reasonably accurately, as tested with self-avoiding random walk (SAW) computer simulations $[17,18]$, and also predicts a significant maximum of the interaction potential. For a suspension of colloidal spheres between the plates, repulsion due to accumulation is a significant effect which is shown using analytical theory to third order in colloid concentration [19], by computer simulation [20] and recently by density functional theory $[21,22]$. Oscillations in the polymer concentration profile around the bulk polymer concentration (that lead to a repulsion) for a polymer solution near a wall have been found by using self-consistent mean-field calculations [23] and by SAW simulations $[17,18]$. However, these oscillations have amplitudes less than a factor of 0.01 of the bulk polymer concentration and the resulting repulsion is therefore extremely weak.

In this communication the potential interaction curve between two parallel plates due to polymers with excluded 
volume is calculated using the adsorption method $[24,25]$ in a simple, convenient fashion. With respect to depletion type of interactions, calculations of the attraction between colloids as induced by non-adsorbing hard rods using the adsorption method has proven to be successful in previous work $[26,27]$. Recently, the adsorption method was used to calculate the interaction between parallel flat plates, between two spheres [3], and between a sphere and a plate [28] for ideal polymer chains. The fact that this approach can be extended to excluded-volume polymers (or real polymers) is due to the developments that have been made in the renormalization group (RG) theory of polymers. The RG theory (Schäfer [29]) provides an accurate expression for the osmotic pressure of a polymer solution that gives the relevant thermodynamic properties required when applying the adsorption method to real polymers.

\section{Theoretical section}

The adsorption method follows from the generalised Gibbs equation [24-26] and leads to the following expression for the interaction potential $W(h)$ :

$$
W(h)=\int_{-\infty}^{\mu}[\Gamma(\infty)-\Gamma(h)] \mathrm{d} \mu^{\prime}
$$

where $\mu$ is the chemical potential of the polymer solution, and $\Gamma(h)$ is the adsorbed amount when the plates are separated at a distance $h$. The adsorption itself is the integral over the local polymer segment concentration profile between the parallel plates $n(x)$ :

$$
\Gamma(h)=\int_{0}^{h} \mathrm{~d} x\left[n(x)-n_{\mathrm{b}}\right]
$$

with $n_{\mathrm{b}}$ the bulk concentration of polymer segments. The bulk polymer segment concentration $n_{\mathrm{b}}$ is here defined as $N\left(3 / 4 \pi R_{\mathrm{g}}^{3}\right) \phi_{\mathrm{p}}$, with $\phi_{\mathrm{p}}$ the relative polymer concentration (三1 at the overlap concentration) and $N$ the number of polymer segments per chain. de Gennes [30] has solved the (Edwards) diffusion equation [31,32] for polymer chains in a gradient field with a self-consistent potential and found a simple equation that describes the polymer segment concentration of a polymer solution near a single wall in a semi-dilute solution of polymers with excluded volume:

$$
n(x)=n_{\mathrm{b}} \tanh ^{2}\left(\frac{x}{\xi}\right),
$$

where $\xi$ is the density correlation length, which thus directly determines the range of the single wall profile. This profile also describes self-consistent mean-field lattice calculations very well [23]. In order to introduce the correct scaling of the correlation length with polymer concentration, we use results from the RG theory [29]. Alternatively, it is possible to derive a profile with the right scaling exponents using the Widom approach [33], as suggested by de Gennes [34]. This approach may lead to a perceptibly better profile but is computationally more involved.

In semi-dilute polymer solutions it is known that the osmotic compressibility is directly related to the density correlation function by the Ornstein-Zernike equation in the thermodynamic limit (wave vector $\rightarrow 0$ ) [30]:

$$
k T \frac{\partial n_{\mathrm{b}}}{\partial \Pi}=n_{\mathrm{b}} \xi^{3},
$$

where $\Pi$ is the osmotic pressure of the polymer solution for which there is a very accurate expression from the RG theory [29]:

$$
\frac{\Pi}{k T}=n_{\mathrm{p}}+5.505 n_{\mathrm{p}}^{2} R_{\mathrm{g}}^{3}\left(\frac{1+2.509 n^{*}+1.360 n^{* 2}}{1+0.596 n^{*}}\right)^{0.309}
$$

where $n^{*}$ equals $3.584 n_{\mathrm{b}} R_{\mathrm{g}}^{3} / N$, and $n_{\mathrm{p}}$ is the bulk polymer concentration $n_{\mathrm{b}} / N$. Further, the density correlation length is defined as $R_{\mathrm{g}}$ at infinite dilution $[29,30,32]$. Consequently, it follows from equations (4) and (5) that

$\frac{\xi}{R_{\mathrm{g}}}=\left(\frac{1}{1+n^{*}+3.073 n^{* 2}\left[\frac{1+3.80 n^{*}+5.67 n^{* 2}}{1+1.73 n^{*}}\right]^{0.309}}\right)^{1 / 3}$

giving $\xi=R_{\mathrm{g}}$ for $n_{\mathrm{b}} \rightarrow 0, \xi \rightarrow 0$ for $n_{\mathrm{b}} \rightarrow \infty$, and the "right" scaling relation for the semi-dilute regime: $\xi \sim$ $n_{\mathrm{b}}^{-0.7697}$. The results of equation (6) agree with smallangle scattering experiments on the correlation length of polystyrene in toluene [35].

Next it is proposed that the polymer concentration profile between two parallel hard plates can be expressed as the product function of the individual profiles of equation (3):

$$
n(x)=n_{\mathrm{b}} \tanh ^{2}\left(\frac{x}{\xi}\right) \tanh ^{2}\left(\frac{h-x}{\xi}\right),
$$

assuming that the superposition approximation gives a good description of the concentration profiles between two particles, as was demonstrated for ideal polymer chains [3, $28]$.

\section{Results and discussion}

To test the superposition approximation, the results of equation (7) (using (6) for the density correlation length) are compared with the SAW simulation results, of which several results were performed by E.J. Meijer which were 


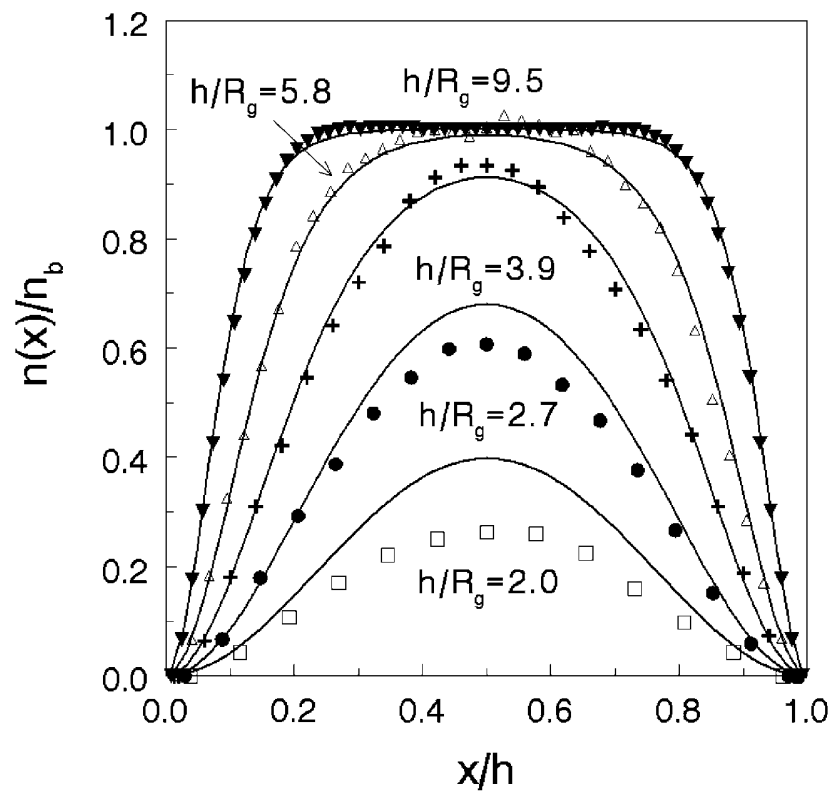

Fig. 1. Relative polymer concentration between two parallel flat plates as a function of the relative position $x / h$ for $\phi_{\mathrm{p}}=0.30$. The full curves are the predictions according to equation (7) (using Eq. (6)). The symbols correspond to the SAW computer simulations.

published in references [17] and [18], and which were made available for us. In Figure $1 \mathrm{SAW}$ results are shown (symbols) for $\phi_{\mathrm{p}}=0.30$ for various relative plate separation distances $h / R_{\mathrm{g}}$, as indicated. The full curves refer to equation (7) using the result for the correlation length of equation (6) from the RG theory, and they match reasonably well with the simulation results. The differences between the simulation results and the product function that occurs for small $h / R_{\mathrm{g}}$ are comparable to those found for ideal polymer chains [3]. For depletion type of interactions the deviation due to the product function approximation at small $h / R_{\mathrm{g}}$ will not have a large effect on the results for the interaction potential since the product function only has a significant relative deviation when the polymer concentration between the plates approaches zero.

Equations (6) and (7) are the ingredients for equation (2) to calculate the adsorption of polymer segments with excluded-volume interaction between two parallel plates. The interaction potential now follows from equation (1). It is convenient to use the thermodynamic relation

$$
\mathrm{d} \mu=\frac{1}{n_{\mathrm{b}}} \mathrm{d} \Pi
$$

from which it follows that equation (1) can be rewritten as

$$
W(h)=\int_{0}^{n_{\mathrm{b}}}\left[\Gamma\left(\infty, n_{\mathrm{b}}^{\prime}\right)-\Gamma\left(h, n_{\mathrm{b}}^{\prime}\right)\right]\left(\frac{1}{n_{\mathrm{b}}^{\prime}} \frac{\partial \Pi}{\partial n_{\mathrm{b}}^{\prime}}\right) \mathrm{d} n_{\mathrm{b}}^{\prime} .
$$
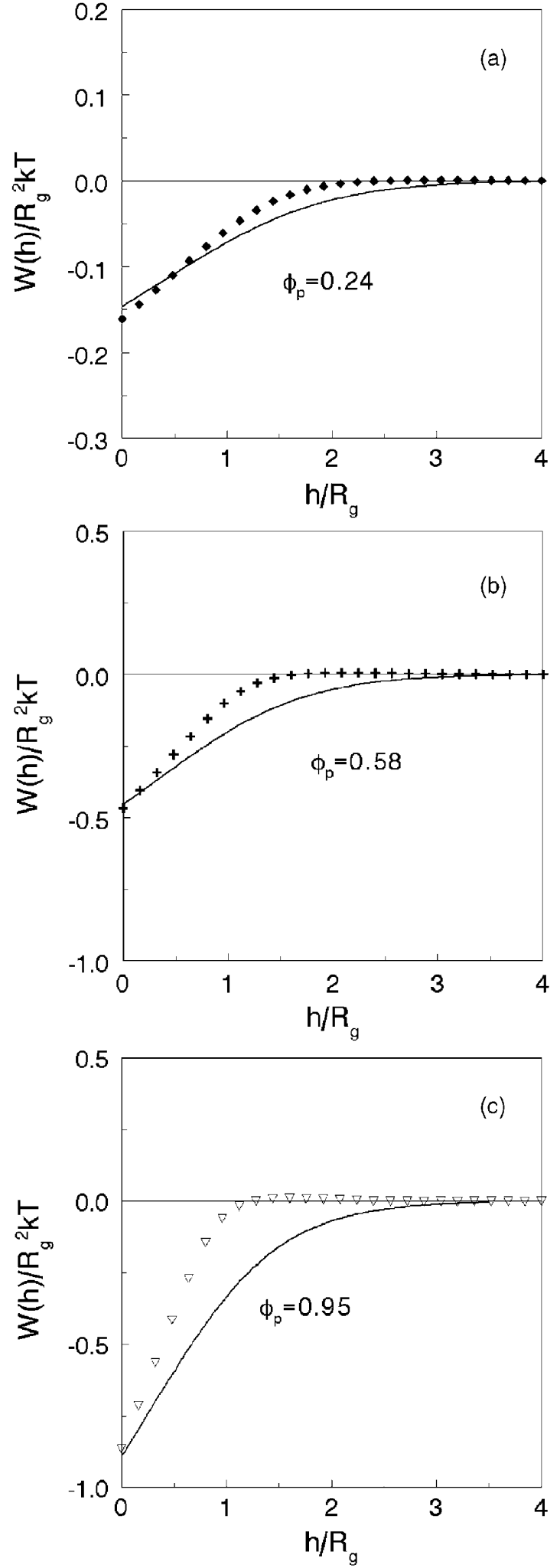

Fig. 2. Interaction potential between two plates immersed in a polymer solution with excluded-volume interaction between the segments from equation (9) (curves) as a function of the normalised distance between the plates $h / R_{\mathrm{g}}$. The results are given for three polymer concentrations as indicated. The symbols refer to SAW simulation results for identical polymer concentrations. 
This equation, using the earlier results for the adsorption (Eqs. (2), (5), (6) and (7)) thus allows a calculation of the interaction between two parallel plates up to very high polymer concentration in the excluded-volume limit. In Figure 2(a-c) the interaction potential between two plates is plotted, for various polymer concentrations as indicated, as a function of the relative plate separation distance, $h / R_{\mathrm{g}}$. The minima of the potentials as determined by the SAW simulations correspond very well to those from equation (9) for the three polymer concentrations. The agreement between the simulation results and our approach is rather reasonable for $\phi_{\mathrm{p}}=0.24$, as plotted in Figure 2(a). It seems that the SAW results correspond to a slightly smaller range of attraction. This effect becomes increasingly more significant for $\phi_{\mathrm{p}}=0.58$ (Fig. 2(b)), and for $\phi_{\mathrm{p}}=0.95$ (Fig. 2(c)). Probably, the difference can be explained by accumulation effects, which are not accounted for in de Gennes' profile (Eq. (3)). Further, the finding that the potential, as calculated from equation (9), is somewhat longer-ranged compared to the SAW simulations corresponds to earlier results for ideal chains [3]. Those results showed that the range of potential as predicted by the product function is slightly longer-ranged, an effect due to the superposition approximation, as compared to exact results probed in simulations. The results of the method presented here do not exhibit a maximum in the interaction potential, an effect that is only present very weakly in the simulation results.

\section{Conclusions}

The minimum of the interaction potential between two parallel flat plates immersed in a solution with polymer chains with excluded-volume interaction can be calculated reasonably accurately using the adsorption method. The required ingredients for the adsorption method are the adsorption of polymer segments and the osmotic compressibility. The adsorption is the integral over the local polymer concentration profile, which is approximated as the product of the individual profiles near a single wall. The profile near a single wall involves the correlation length, which is calculated using the osmotic compressibility. The approach presented here is reasonably consistent with selfavoiding random walk computer simulations and has the advantage that no "colloid" behaviour of the polymer chains is assumed whatsoever.

This work was supported by the Council for Chemical Sciences of the Netherlands Organization for Scientific Research (NWO/CW) and Unilever Research Vlaardingen. We wish to express our thanks to E.J. Meijer, who provided us with the SAW simulation results. We would like to thank E. Eisenriegler for useful discussions and J.-F. Joanny, for pointing out reference [34] to us. A. Vrij and V.J. Anderson are thanked for useful suggestions and a critical reading of the manuscript.

\section{References}

1. S. Asakura, F. Oosawa, J. Chem. Phys. 22, 1255 (1954).

2. A. Vrij, Pure Appl. Chem. 48, 471 (1976).

3. R. Tuinier, G.A. Vliegenthart, H.N.W. Lekkerkerker, J. Chem. Phys. 113, 10768 (2000).

4. A.P. Gast, C.K. Hall, W.B. Russel, J. Colloid Interface Sci. 96, 251 (1983).

5. H.N.W. Lekkerkerker, W.C.K. Poon, P.N. Pusey, A. Stroobants, P.B. Warren, Europhys. Lett. 20, 559 (1992).

6. E.J. Meijer, D. Frenkel, J. Chem. Phys. 100, 6873 (1994).

7. M. Dijkstra, J. Brader, R. Evans, J. Phys. Condens. Matter 11, 10079 (1999)

8. P.G. de Gennes, C.R. Acad. Sci. B 288, 359 (1979).

9. T. Odijk, Macromolecules 29, 1842 (1996).

10. T. Odijk, J. Chem. Phys. 106, 3402 (1997).

11. E. Eisenriegler, J. Chem. Phys. 113, 5091 (2000).

12. J.F. Joanny, L. Leibler, P.G. de Gennes, J. Polym. Sci. Polym. Phys. 17, 1073 (1979).

13. A.P. Chatterjee, K.S. Schweizer, J. Chem. Phys. 109, 10477 (1998).

14. A.P. Chatterjee, K.S. Schweizer, J. Chem. Phys. 109, 10464 (1998).

15. M. Fuchs, K.S. Schweizer, Europhys. Lett. 51, 621 (2000).

16. N.P. Shusharina, P.G. Khalatur, A.R. Khokhlov, J. Chem. Phys. 113, 7006 (2000).

17. A.A. Louis, P.G. Bolhuis, J.P. Hansen, E.J. Meijer, Phys. Rev. Lett. 85, 2522 (2000).

18. P.G. Bolhuis, A.A. Louis, J.P. Hansen, E.J. Meijer, J. Chem. Phys. 114, 4296 (2001).

19. Y. Mao, M.E. Cates, H.N.W. Lekkerkerker, Physica A 222, 10 (1995).

20. T. Biben, P. Bladon, D. Frenkel, J. Phys. Condens. Matter 8, 10799 (1996).

21. B. Götzelmann, R. Roth, S. Dietrich, M. Dijkstra, R. Evans, Europhys. Lett. 47, 398 (1999).

22. R. Roth, R. Evans, S. Dietrich, Phys. Rev. E 62, 5360 (2000).

23. J. Van der Gucht, N.A.M. Besseling, J. Van Male, M.A. Cohen Stuart, J. Chem. Phys. 113, 2886 (2000).

24. D.G. Hall, J. Chem. Soc. Faraday Trans. 68, 2169 (1972).

25. S.G. Ash, D.H. Everett, C. Radke, J. Chem. Soc. Faraday Trans. 69, 1256 (1973).

26. Y. Mao, P. Bladon, H.N.W. Lekkerkerker, M.E. Cates, Mol. Phys. 92, 1510 (1997).

27. R.P. Sear, Phys. Rev. E 57, 1983 (1998).

28. R. Tuinier, G.A. Vliegenthart, H.N.W. Lekkerkerker, Macromolecules 34, 4636 (2001).

29. L. Schäfer, Excluded Volume Effects in Polymer Solutions (Springer Verlag, Berlin, 1999); see especially Chapts. 1719.

30. P.G. de Gennes, Scaling Concepts in Polymer Physics (Cornell University Press, Ithaca, 1979).

31. S.F. Edwards, K.F. Freed, J. Phys. A 2, 145 (1969).

32. M. Doi, S.F. Edwards, The Theory of Polymer Dynamics (Clarendon Press, Oxford, 1986).

33. J.S. Rowlinson, B. Widom, Molecular Theory of Capillarity (Clarendon Press, Oxford, 1989) sect. 9.4.

34. P.G. de Gennes, Macromolecules 14, 1637 (1981).

35. F. Hamada, S. Kinusaga, H. Hayashi, A. Nakajima, Macromolecules 18, 2290 (1985). 